\title{
Mobile Game-Based Digital Vaccine for Reducing Risk of Lifestyle Diseases
}

\author{
Rema Padman ${ }^{1}$, MS, PhD; Yi-Chin Kato-Lin ${ }^{2}$, MS, PhD; Bhargav SriPrakash ${ }^{3}$, MS Eng; Sross Gupta ${ }^{1}$, MS Info Sys; \\ Palak Narang ${ }^{1}$, MS Info Sys; Preethika Karthikeyan ${ }^{1}$, BSc; Uttara Bharath-Kumar ${ }^{4}$; Pradeep Krishnatray ${ }^{4}$, PhD; \\ Sanjeeta Agnihotri ${ }^{4}$; Bhairavi Prakash ${ }^{5}$; Vasini Varadan ${ }^{6}$
}

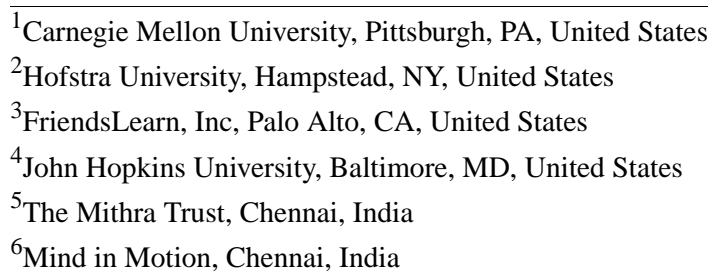

Corresponding Author:

Yi-Chin Kato-Lin, MS, PhD

Hofstra University

134 Hofstra University

Hampstead, NY,

United States

Phone: 4634269

Email: YiChin.Lin@hofstra.edu

\section{Abstract}

Background: There is a rising epidemic of pediatric obesity in the United States and worldwide. While many factors contribute to pediatric overweight and obesity, dietary decisions are a leading cause. Children spend many hours a day playing video games, mostly on mobile devices. Hence, personalized gamification and learnification on mobile devices have great potential to influence children's dietary-lifestyle behaviors during the habit formation stage of early childhood. In fact, video games on mobile devices have become a platform through which children learn in a fun and enjoyable way. While there is some early evidence of the positive impact of neuropsychology-based, cartoon-styled, immersive video games on healthy eating behaviors in children, the mechanisms underlying these improved outcomes are yet to be understood.

Objective: To design appropriate interventions in the game environment for children's behavior formation and change, we need to learn more about the underlying patterns of player behaviors evidenced during gameplay through techniques of machine learning and stochastic optimization. Building on prior descriptive work, this study examines the impact of a diet and lifestyle focused mobile game on children's game play patterns and associate these patterns with their actual food choices using machine learning and statistical models.

Methods: Our dataset was generated from an IRB-approved, informed consent-based randomized controlled trial (RCT) with pre- and post-treatment measurements of almost 100 school children using fooya!, a novel mobile gaming, iOS/Android based App that is being developed as a low-risk and non-invasive "digital vaccine" for lifestyle diseases, for 2 exposures of 20 minutes each. Based on artificial intelligence, neuropsychology and cognitive behavior therapy, fooya! has been shown to deliver positive outcomes with respect to food choices, self-reported dietary choices, and healthy eating intentions. We first model the process of game playing at any level across all students as a discrete, time-homogeneous, first-order Markov chain with multiple states, each representing a status of the game. Process mining identifies distinct patterns in the game sequences and statistical models establish the relationship between game patterns combined with demographic and behavioral data with actual food choices at the end of the game.

Results: We find strong evidence of the positive effect of the mobile game on actual food choices, just after 40 minutes of intervention exposure (T: $2.46 ; \mathrm{C}: 1.10 ; P<.001)$. Analysis of children's play patterns shows significant variations in game play mechanics among players. Regression analyses further reveal that more engaged, dynamic, and strategic game play patterns are associated with better actual food choices. 
Conclusions: This study adds to the growing body of evidence that learning about healthy eating in a fun and exciting way via mobile games, acting as Digital Vaccines, can positively impact children's actual food choices. While promising, additional RCTs in varied settings and deeper analysis of the resulting data are needed to confirm Digital Vaccines' potential to reduce the long-term risk of nutrition related non-communicable diseases such as diabetes and cardiovascular disease, as well as health risks from the double burden of overweight vs malnutrition and under-nutrition by educating children regarding healthy lifestyle choices using mobile games.

(iproc 2018;4(2):e11790) doi: $\underline{10.2196 / 11790}$

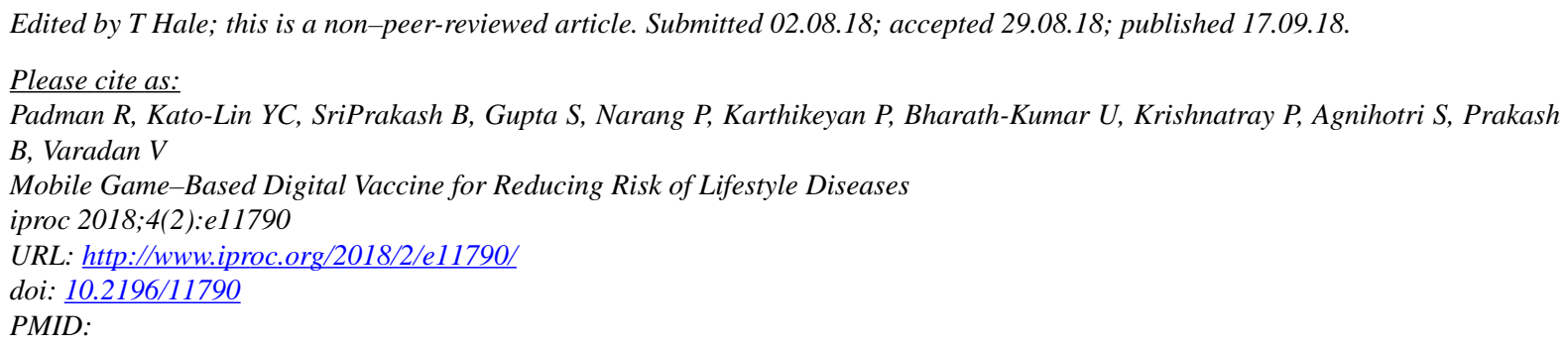

(CRema Padman, Yi-Chin Kato-Lin, Bhargav SriPrakash, Sross Gupta, Palak Narang, Preethika Karthikeyan, Uttara Bharath-Kumar, Pradeep Krishnatray, Sanjeeta Agnihotri, Bhairavi Prakash, Vasini Varadan. Originally published in Iproceedings (http://www.iproc.org), 17.09.2018. This is an open-access article distributed under the terms of the Creative Commons Attribution License (https://creativecommons.org/licenses/by/4.0/), which permits unrestricted use, distribution, and reproduction in any medium, provided the original work, first published in Iproceedings, is properly cited. The complete bibliographic information, a link to the original publication on http://www.iproc.org/, as well as this copyright and license information must be included. 